\title{
Perfil da fluência verbal em indivíduos com a Doença de Parkinson
}

\author{
Renata Lucca de Souza*, Maria Cristina de Almeida Freitas Cardoso*
}

\section{Resumo}

A fluência verbal é uma função cognitiva que incorpora dados motores relacionados ao tempo de fonação e da velocidade de fala, assim como dos aspectos da efetividade da linguagem, a partir de componentes linguísticos, semânticos e fonológicos, no estabelecimento do seu perfil. Este estudo tem por objetivo verificar a fluência verbal de um grupo de indivíduos com a doença de Parkinson. Trata-se de um estudo descritivo, transversal, observacional e quantitativo cujo fator em estudo é o perfil da fluência verbal e o seu desfecho, doença de Parkinson. Compõe-se de uma amostra de 18 indivíduos de ambos os sexos (nove muIheres e nove homens) com idades de 48 a 78 anos, sendo a média etária de 65,5 \pm 8,4 anos. Foram verificados o tempo de fonação, a eficiência glótica e os testes de fluência verbal. Na avaliação dos tempos máximos de fonação (TMF), verifica-se a média para vogais de $16,84 \mathrm{~s}$. A média da relação s/z é de 0,84 e do tempo de leitura por minuto de 130 pals./min. À fluência verbal semântica confere-se a média de 16 pals./ min. e para a fluência verbal fonológica a média de 17 pals./min. Conclui-se que os TMF encontram-se adequados às variáveis idade e gênero. A fluência verbal semântica está abaixo do encontrado na literatura e acima para com a fluência verbal fonológica. Reforça-se a correlação entre a fluência verbal e o tempo de escolaridade, assim como, a preservação cognitiva do grupo.

Palavras-chave: Indivíduos com Parkinson. Semântica. Testes de Linguagem.

\section{Introdução}

A Doença de Parkinson (DP) é um dos distúrbios do movimento caracterizado por movimentos involuntários e/ou perda do controle ou da eficiência em movimentos voluntários que compromete a qualidade de vida dos seus portadores, cuja incidência é variável, mas que se eleva no envelhecimento (MUNHOZ et al., 2012; CHANA et al., 2013). Trata-se de uma doença degenerativa progressiva decorrente da morte de células da substância negra compacta e de outros núcleos pigmentados do tronco encefálico, ela é caracterizada por um esgotamento

* Bacharel em fonoaudiologia pelo Centro Universitário Metodista do IPA, Porto Alegre (RS). Email: renatalucca@hotmail.com.

** Fonoaudióloga Doutora em Gerontologia Biomédica, Professora Adjunto I da Universidade Federal de Ciências da Saúde de Porto Alegre - UFCSPA. Endereço: Av. Eduardo Prado, 695 casa 37, Porto Alegre/RS, CEP 91751-000. E-mail: mccardoso@via-rs.net.

$\rightarrow$ http://dx.doi.org/10.5335/rbceh.2014.3237

Recebido em: 31/05/2013. Aprovado em: 14/07/2014 
seletivo do neurotransmissor dopamina. Dessa forma, acomete principalmente o sistema motor e acaba por se refletir em alterações comunicativas (AZEVEDO; CARDOSO, 2009).

$\mathrm{Na}$ literatura, são referenciados transtornos fonoaudiológicos observados na DP que se compreendem por alterações nos sistemas pneumofonoarticulatórios caracterizados por intensidade vocal reduzida, articulação imprecisa, alteração da velocidade da fala, jatos de fala e monotonia de frequência e de intensidade (MOURÃO, 2010).

As variáveis acústicas de frequência e intensidade alteradas na fala do indivíduo com a DP refletem as mudanças fisiológicas e anatômicas causadas pela perda das células dopaminérgicas no sistema nervoso central. Ao mesmo tempo, a perda do input dopaminérgico no corpo estriado e a consequente desregulação dos gânglios basais produzem os déficits motores que comprometem três subsistemas relacionados ao controle motor da fala (respiratório, fonatório e articulatório), em que a alteração em um desses sistemas irá afetar a voz e a coordenação da fala (VITORINO; HOMEM, 2001; MARTINEZ-SANCHES, 2010).

Os déficits na força muscular respiratória, comuns na DP, decorrem da rigidez e do encurtamento muscular, os quais se refletem no menor suporte respiratório à emissão vocal e, por consequência, na intensidade vocal e nos tempos máximos de fonação. A postura corporal do parkinsoniano em flexão (flexão da cabeça e dos braços, hipercifose torácica e a prostração de ombros) altera especialmente as colunas torácica e lombar, gerando uma desvantagem biomecânica à atividade da musculatura respiratória, ocasionando menor capacidade de expansão pulmonar, redução dos volumes pulmonares e déficit na excursão diafragmática (FERREIRA, 2008; OLANOW; STERN; SETHI, 2009).

A imprecisão ou a não exatidão dos pontos articulatórios observada na fala dos indivíduos com a DP, é referida como "enrolada", comprometendo o entendimento da mensagem, estando relacionada à rigidez da musculatura facial (LIMONGI, 2001).

A fluência verbal é a capacidade de produzir uma fala espontaneamente fluída, sem excessivas pausas nem falhas na busca das palavras, estando associada ao ritmo, à velocidade, à entonação e às intenções comunicativas (CRENITTE; LOPES-HERRERA, 2009; SOUZA; CARDOSO, 2013).

A disfluência neurológica, encontrada na DP, é caracterizada por uma desorganização da fala ou da linguagem, devido aos transtornos neurológicos, na qual se observa a ocorrência de ritmo alterado, sons distorcidos, velocidade da fala acelerada ou mais lenta do que o normal e uma entonação monótona (JAKUBOVICZ, 1997; VITORINO; HOMEM, 2001). Já na prosódia, o significado claro das palavras tanto em unidades fonológicas, lexicais, morfológicas, sintáticas e semânticas da linguagem, mostra-se alterado devido a não flutuações da frequência, aparecendo alterações na capacidade para produzir entonação emocional e, em estágios avançados, para decodificação da linguagem dos outros (MARTINEZ-SANCHES, 2010). 
Os processos cognitivo-linguísticos semânticos e fonológicos, verificados através dos testes de fluência verbal fornecem parâmetros sobre a efetividade da linguagem (ANDRADE, 2000) e, por ser uma tarefa complexa, envolve o funcionamento do encéfalo (SANTOS, 2006).

Os testes de fluência verbal estão inseridos numa série de testes neuropsicológicos, baseando-se no desempenho de indivíduos normais, comparados a pacientes com doenças previamente estabelecidas, relacionados a déficits de desempenho e a área comprometida (RODRIGUES; YAMASHITA; CHIAPPETTA, 2008). Essas provas, tanto a semântica quanto a fonológica, são realizadas para verificar a capacidade de armazenamento semântico, a habilidade de recuperação da informação e do planejamento das funções executivas (BUTMAN et al., 2000).

$\mathrm{Na}$ literatura encontram-se referencias quanto aos comprometimentos motores dos parkinsonianos, com diferenças estatísticas estabelecidas. Os aspectos cognitivos são referidos como presentes, sendo a depressão como uma ocorrência prevalente, o que pode interferir na execução cognitiva (PIOVEZAN et al., 2007; BRUNA et al., 2008).

Este estudo tem como objetivo verificar a fluência verbal de um grupo de indivíduos com a DP, considerando a necessidade de verificação da deteriorização cognitiva junto aos parkinsonianos.

\section{Método}

Estudo descritivo de caráter transversal, observacional e quantitativo, cujo fator é o perfil da fluência verbal e o seu desfecho, a DP. Esta pesquisa foi realizada com indivíduos com a DP que fazem parte da Associação Parkinson do Rio Grande do Sul - Apars, cujas atividades ocorrem na Associação Médica do Rio Grande do Sul - Amrigs, em Porto Alegre/RS e no Centro Universitário Metodista do IPA. Esta investigação foi aprovada pelo Comitê de Ética em Pesquisa - CEP - do Centro Universitário Metodista do IPA de Porto Alegre/RS, sob o protocolo número 106/2011. Todos os participantes assinaram o termo de conhecimento livre e esclarecido, conforme o previsto em estudos com humanos.

Trata-se de um grupo censo, de conveniência, composto por 18 indivíduos com diagnóstico de Doença de Parkinson. Os critérios de inclusão foram terem diagnóstico estabelecido e aceitarem participar do estudo.

Os 18 indivíduos com DP que participaram deste estudo foram de ambos os sexos (nove femininos e nove masculinos) com idade média de 65,5 anos.

\section{Procedimentos}

Para a realização do trabalho investigativo foi utilizada uma breve anamnese direcionada aos aspectos relevantes da DP, na qual foi anotado o estágio de acometimento da doença, segundo a "Escala de Estadiamento de Hoehn e Yahr" (HOEHN; YAHR, 1967), tendo sido essa separada em cinco estágios, I à III, leve a moderado e IV e $\mathrm{V}$ graves e verificada a idade, o sexo, o tempo de estudo e a data do diagnóstico da DP.

O tempo de estudo ou grau de escolaridade permitiu dividir os participantes em dois grupos, sendo o grupo 1 (G1) com 
o tempo de escolaridade compreendido entre 8 e 13 anos e o grupo 2 (G2) com 15 anos ou mais de escolaridade.

Após, foi realizada uma avaliação clínica da fluência verbal, em sala anexa ao local das atividades de grupo da Apars pela pesquisadora assistente. A avaliação clínica engloba a respiração e a fonação (com a devida cronometragem dos tempos máximos de fonação - TMF, a relação entre os fones /s/ e/z/ e o tempo de leitura de um texto), gravação e cronometragem do tempo para a fluência verbal, através da solicitação da nomeação de palavras iniciadas com o fonema /a/ para a fluência verbal fonológica e palavras da categoria semântica de animais na fluência verbal semântica. Cada solicitação foi cronometrada no tempo de um minuto, seguindo o protocolo proposto por Rigon (2010).

Para a correlação entre os níveis de comprometimento da DP e os resultados da fluência verbal semântica e fonológica também foram organizados dois grupos, ou seja, grupo A (G.A) para os estágios I e II e o grupo B (G.B), agrupando os participantes dos estágios III e IV.

Para comparação das variáveis, categóricas entre as faixas de tempo de estudo e os estágios da doença foram utilizados os testes Qui-Quadrado ou exato de Fisher. Para as variáveis numéricas entre dois grupos (as faixas de tempo de estudo, os estágios da doença e os gêneros) foi utilizado o teste de Mann-Whitney, devido à ausência de distribuição normal das variáveis. Para analisar a relação entre a idade e as variáveis numéricas, foi utilizado o coeficiente de correlação de Spearman devido à ausên- cia de distribuição normal das variáveis. O nível de significância adotado para os testes estatísticos foi de $p<005$.

\section{Resultados}

$\mathrm{Na}$ avaliação dos tempos máximos de fonação (TMF), verificou-se para o fonema /a/ uma média de $16,19 \mathrm{~s}$, para o fonema /i / média de 17,03s, para o /u/ média de $17,3 \mathrm{~s}$. A média total das vogais foi de $16,84 \mathrm{~s}$, tendo sido encontrada para o sexo feminino a média de $14,56 \mathrm{~s}$ e masculino 19,13s. O TMF do fonema /s/ teve a média de $13,08 \mathrm{~s}$ e do fonema / $\mathrm{z} /$ a média de $15,37 \mathrm{~s}$. A média da relação /s/ /z/ foi de 0,84s. A comparação entre essas médias encontradas encontram-se no Gráfico 1.

Gráfico 1 - Comparação entre as médias dos tempos máximos de fonação

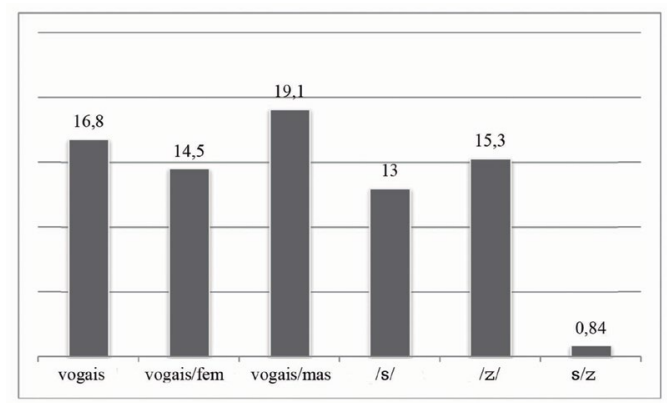

Nota: *Tempos Máximos de Fonação em segundos.

A verificação do tempo da leitura por minuto mostrou uma média de 130,28 pal. Na fluência verbal semântica, os participantes apresentaram uma média de 16,39 pals./min. e, na fluência verbal fonológica uma média de 17,28 pals./ min., abaixo do referenciado para adultos hígidos. 
A correlação entre os dados da flu- para o G2 19,33 pals./min. Essas médias ência verbal semântica e o tempo de evidenciaram diferença entre os graus escolaridade foi de: G1 uma média de de escolaridade, porém a correlação 14,7 pals./min.; G2 uma média de 18 mostrou resultados não significativos pals./min. A análise da fluência verbal $\quad(p=0,215$ e $p=0,184$, respectivamente). fonológica e o tempo de estudo foi para Esses dados podem ser visualizados na o G1 uma média de 15,22 pals./min. e Tabela 1.

Tabela 1 - Comparação das variáveis numéricas entre escolaridade

\begin{tabular}{|c|c|c|c|c|}
\hline $\begin{array}{c}\text { Escolaridade } \\
\text { (anos de estudos) }\end{array}$ & $n$ & Variável & $\begin{array}{l}\text { Média/ Desvio } \\
\text { Padrão }\end{array}$ & $p$ \\
\hline $8-13$ & 9 & $\begin{array}{l}\text { Idade } \\
\text { TMF /a/ } \\
\text { TMF /i/ } \\
\text { TMF /u/ } \\
\text { TMF /s/ } \\
\text { TMF /z/ } \\
\text { Relação /s/ /z/ } \\
\text { Leitura - número de palavras por minuto } \\
\text { Fluência verbal semântica } \\
\text { Fluência verbal fonológica }\end{array}$ & $\begin{array}{r}6822 \pm 9,09 \\
120 \pm 5,73 \\
117 \pm 6,94 \\
135 \pm 6,50 \\
111 \pm 4,87 \\
124 \pm 5,49 \\
090 \pm 0,16 \\
118 \pm 26,81 \\
14 \pm 6,30 \\
15 \pm 4,84\end{array}$ & $\begin{array}{l}0,101 \\
0,015 \\
0,070 \\
0,024 \\
0,508 \\
0,310 \\
0,251 \\
0,133 \\
0,215 \\
0,184\end{array}$ \\
\hline$\geq 15$ anos & 9 & $\begin{array}{l}\text { ldade } \\
\text { TMF /a/ } \\
\text { TMF /i/ } \\
\text { TMF /u/ } \\
\text { TMF /s/ } \\
\text { TMF /z/ } \\
\text { Relação /s/ /z/ } \\
\text { Leitura - número de palavras por minuto } \\
\text { Fluência verbal semântica } \\
\text { Fluência verbal fonológica }\end{array}$ & $\begin{array}{r}62,7 \pm 7,28 \\
203 \pm 6,3 \\
203 \pm 7,4 \\
210 \pm 7,8 \\
150 \pm 10,9 \\
183 \pm 10,4 \\
08 \pm 0,20 \\
142 \pm 34,7 \\
18 \pm 5,68 \\
19 \pm 7,42\end{array}$ & $\begin{array}{l}0,101 \\
0,015 \\
0,070 \\
0,024 \\
0,508 \\
0,310 \\
0,251 \\
0,133 \\
0,215 \\
0,184\end{array}$ \\
\hline
\end{tabular}

Nota: Valor $p$ refere-se ao teste de Mann-Whitney para comparação dos valores entre escolaridade; TMF - tempos máximos de fonação em segundos. 
Na correlação dos níveis de compro- pals./min. e o G.B de 19 pals./min. (dp \pm metimento da DP com os resultados da 7,1 pals./min.). As correlações entre os avaliação da fluência verbal semântica o G.A (Escala de estadiamento de Hoehn e Yahr I e II) obteve uma média de 14,8 pals./min. e para o G.B (Escala III e IV) 18,3 pals./min. Quanto à fluência verbal fonológica, o G.A obteve uma média de 16 grupos de comprometimento da DP com a fluência verbal semântica e a fluência verbal fonológica não foram significativos ( $p=0,109$ e $p=0,305$ respectivamente). Os dados dessas análises comparativas podem ser conferidos na Tabela 2 .

Tabela 2 - Comparação das variáveis numéricas entre estágios da doença

\begin{tabular}{|c|c|c|c|c|}
\hline Estágios DP & $n$ & Variável & Média Padrão - dp & $p^{*}$ \\
\hline$|-| \mid$ & 10 & $\begin{array}{l}\text { Idade (anos) } \\
\text { TMF /a/ } \\
\text { TMF /i/ } \\
\text { TMF /u/ } \\
\text { TMF /s/ } \\
\text { TMF /z/ } \\
\text { Relação /s/z } \\
\text { Leitura - número de palavras por minuto } \\
\text { Fluência Verbal Semântica } \\
\text { Fluência Verbal fonológica }\end{array}$ & $\begin{array}{r}67,40 \pm 8,29 \\
15 \pm 7,64 \\
15 \pm 7,11 \\
15 \pm 6,99 \\
14 \pm 10,40 \\
15 \pm 9,37 \\
088 \pm 0,19 \\
119 \pm 24,31 \\
148 \pm 7.07 \\
159 \pm 5,34\end{array}$ & $\begin{array}{l}0,196 \\
0,424 \\
0,477 \\
0,328 \\
0,859 \\
0,929 \\
0,398 \\
0,168 \\
0,109 \\
0,305\end{array}$ \\
\hline III-IV & 8 & $\begin{array}{l}\text { Idade } \\
\text { TMF /a/ } \\
\text { TMF /i/ } \\
\text { TMF /u/ } \\
\text { TMF /s/ } \\
\text { TMF /z/ } \\
\text { Relação /s/ /z/ } \\
\text { Leitura - número de palavras por minuto } \\
\text { Fluência Verbal Semântica } \\
\text { Fluência Verbal Fonológica }\end{array}$ & $\begin{array}{r}63,13 \pm 8,61 \\
17 \pm 6,94 \\
18 \pm 8,66 \\
19 \pm 8,97 \\
11 \pm 5,52 \\
15 \pm 8,25 \\
081 \pm 0,18 \\
143 \pm 37,67 \\
18 \pm 4,07 \\
19 \pm 7,15\end{array}$ & $\begin{array}{l}0,196 \\
0,424 \\
0,477 \\
0,328 \\
0,859 \\
0,929 \\
0,398 \\
0,168 \\
0,109 \\
0,305\end{array}$ \\
\hline
\end{tabular}

Nota: * $p$ referente ao teste de Mann-Whitney para comparação dos valores entre estágios da doença; TMF - tempos máximos de fonação em segundos. 
Ao comparar o grau de escolaridade com o estágio da DP, verificou-se para os participantes, cujos estágios compreendiam os níveis I e II, o resultado de $77,78 \%$ para aqueles com 8 a 13 anos de estudo e, $33,33 \%$ para 15 anos ou mais. Para os estágios III e IV, encontrou-se o resultado de $22,22 \%$ para 8 a 13 anos de estudo e, $66,67 \%$ para com 15 anos ou mais de estudo.
Em relação à correlação da idade dos participantes e as demais variáveis numéricas, os resultados apontam uma correlação significativa entre a idade e a fluência verbal fonológica ( $\mathrm{r}=0.554$, $p=0.017$ ), ou seja, quanto mais a idade, menor a fluência verbal fonológica. Esses dados podem ser analisados na Tabela 3 e Gráfico 2.

Tabela 3 - Correlações entre idade e outras variáveis numérica para 18 sujeitos

\begin{tabular}{|c|c|c|c|c|c|c|c|}
\hline & & TMF /a/ & TMF /i/ & TMF /u/ & TMF /s/ & TMF /z/ & Relação s/z \\
\hline \multirow{2}{*}{ Idade } & $r$ & -0.12015 & -0.12532 & -0.18333 & -0.07043 & -0.09425 & 0.17574 \\
\hline & $p$ & 0.6349 & 0.6202 & 0.4665 & 0.7812 & 0.7099 & 0.4855 \\
\hline & & $\begin{array}{l}\text { Leitura - número } \\
\text { de palavras por } \\
\text { minuto }\end{array}$ & $\begin{array}{l}\text { Fluência Verbal } \\
\text { Semântica }\end{array}$ & $\begin{array}{l}\text { Fluência Verbal } \\
\text { Fonológica }\end{array}$ & & & \\
\hline \multirow{2}{*}{ Idade } & $\mathrm{R}$ & -0.20788 & -0.29886 & -0.55434 & & & \\
\hline & $P$ & 0.4078 & 0.2283 & 0.0170 & & & \\
\hline
\end{tabular}

Nota: r=coeficiente de correlação de Spearman; TMF - tempos máximos de fonação.

Gráfico 2 - Correlação entre a fluência verbal fonológica e a idade dos participantes

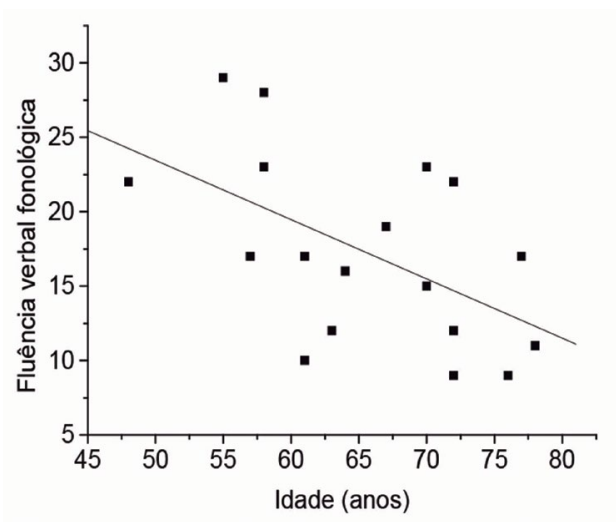

\section{Discussão}

No presente estudo, a média de idade dos indivíduos com a DP é de 65,5 anos, tendo o mais novo 48 anos e o mais velho 78 anos, estando esses dados entre a faixa etária de diagnóstico da doença conforme Menezes e Teive (1996). Em relação ao sexo, encontra-se na literatura que a DP afeta aproximadamente o mesmo número de homens e de mulheres, não havendo cromossomos sexuais envolvidos (LIMONGI, 2001).

Com relação aos gêneros e aos TMF, os participantes apresentaram os seguintes resultados: para o sexo feminino, tem-se o tempo médio de $14,56 \mathrm{~s}$ e, para o sexo masculino o tempo de $19,13 \mathrm{~s}$. 
Considerando as variáveis verificadas, a partir de um estudo realizado na cidade de São Paulo, observa-se que os valores dos TMF esperados para a idade acima de sessenta anos é em torno de $14 \mathrm{~s}$ para mulheres e em torno de $20 \mathrm{~s}$ para homens (BEHLAU; PONTES, 1995).

Em estudo com um grupo de idosos gaúchos sem queixas de qualquer transtorno, a maioria do sexo feminino, encontrou-se os TMF com uma média geral de 17,9 s (RIGON, 2010). Conforme pesquisa, as médias de TMF para os sexos masculino e feminino portadores da DP foram, respectivamente, de $10 \mathrm{~s}$ e 7 s (DIAS; LIMONGI, 2003). Com base nesses dados, observa-se que as médias de TMF, no presente estudo, encontram-se dentro do esperado para os sexos feminino e masculino, com a média geral (de 16,85s), aproximada aos dados dos idosos gaúchos e acima do encontrado para com os portadores de DP (BEHLAU; PONTES, 1995; DIAS; LIMONGI, 2003; RIGON, 2010).

Para com a variável numérica da relação /s/ / z/, no presente estudo, tem-se para o gênero feminino uma média de $0,88 \mathrm{~s}$ e para o gênero masculino 0,80 s. Esses resultados são apontados por Behlau et al. (2001), como de uma hipercontração das pregas vocais à fonação. Rigon (2010) computou a média para um grupo de idosos gaúchos sem patologia em $1,2 \mathrm{~s}$, considerado sugestivo de falta de coaptação correta das pregas vocais para a fonação, conforme a literatura (BEHLAU et al., 2001). Em um estudo realizado com portadores de $\mathrm{DP}$ por $\mathrm{Pa}-$ lermo et al. (2009), tem-se que 96\% dos participantes com DP apresentam uma falta de eficiência glótica e, cerca de $12 \%$ mostraram-se com hipercontração das pregas vocais.

Os resultados obtidos no presente estudo para a relação /s/ /z/ contrariam os encontrados na literatura para com a população de idosos gaúchos (RIGON, 2010), mas corroboram os dados referentes aos indivíduos com a DP(DIAS; LIMONGI, 2003; PALERMO et al., 2009), visto que a hipercontração pode ser explicada pela redução da expansibilidade torácica, frequentemente encontrada em indivíduos com a DP, e tanto essa como o fechamento glótico incompleto afetam a intensidade vocal e interferem negativamente na comunicação (ROSA; CIELO; CECHELLA, 2009).

Em estudo realizado com indivíduos com a DP encontram-se, para o sexo feminino, valores, pré-terapia, da relação $/ \mathrm{s} / \mathrm{z} /$ maiores que 1,2 , indicando alteração da eficiência glótica por falta de coaptação correta das pregas vocais, pois o grupo estudado apresentava comprometimento do suporte respiratório e sugere que tal dado explica a instabilidade vocal (VICCO; SANTOS; GONÇALVES, 2009).

Para o tempo de leitura por minuto, tem-se uma média geral considerada no limite do padrão de normalidade para com os participantes desse estudo, porém, ao se distinguir os gêneros, encontra-se para o feminino uma média de $127,33 \mathrm{pals} . / \mathrm{min}$., o qual é considerado abaixo da média esperada. A literatura assevera que, para o tempo abaixo de 130 pals./min., os ouvintes consideram o falante muito lento e, acima de 180 pals./min. inadequadamente rápido (BEHLAU; PONTES, 1995). Em Rigon (2010) tem-se a média do tempo de leitura de $141,6 \mathrm{pal} . / \mathrm{min}$. 
Na correlação da fluência verbal semântica quando comparada com o grau de escolaridade, verifica-se a ocorrência de uma pequena diferença para com indivíduos com a DP com oito a 13 anos de escolaridade, pois o grupo obteve uma média de 14 pals./min., enquanto o grupo dos portadores com 15 anos de escolaridade, a média foi de $18 \mathrm{pals} . / \mathrm{min}$.

No estudo realizado com participantes idosos encontra-se que, na faixa etária entre 60-80 anos com 5 a 8 anos de escolaridade apresentam uma média de 15 pals./min. e os idosos entre 60-80 anos com 12 anos ou mais de escolaridade, 19 pals./min.(RODRIGUES; YAMASHITA; CHIAPPETTA, 2008). No estudo com um grupo de idosos gaúchos, encontrou-se a média de tempo de estudo de 13 anos, tendo a categoria de fluência verbal semântica uma média de 19,9 pals./min. (RIGON, 2010). Essas médias verificadas na literatura, quanto ao número de palavras evocadas por minuto, estão acima do encontrado em participantes do presente estudo.

Quanto à fluência verbal fonológica os participantes apresentaram uma pequena diferença entre o grau de escolaridade, ou seja, para o grupo de oito a 13 anos de escolaridade, tem-se uma média de 15 pals./min. e para os portadores do grupo com 15 anos ou mais de escolaridade, uma média de 19 pals./min.

Ao comparar esses dados estabelecidos de fluência verbal fonológica ao verificado, quanto ao tempo de escolaridade e a literatura, encontra-se que idosos com idade entre sessenta e oitenta anos que tenha de cinco a oito anos de escolaridade, apresentaram uma média de 9,5 pals./min. e os idosos com 12 anos ou mais de escolaridade têm uma média de 13 pals./min.(RODRIGUES; YAMASHITA; CHIAPPETTA, 2008). A média de palavras por minuto na fluência verbal fonológica em idosos sem queixas de transtornos é de 15,5 pals./ min. (RIGON, 2010). Os dados coletados em referência a essa variável mostram-se acima do encontrado na literatura. Tal divergência pode ser sugestiva da preservação do lobo frontal dos participantes avaliados, com boa performance para com as funções executivas.

Ao correlacionar a idade dos participantes da DP com o resultado da fluência verbal fonológica, verifica-se correlação significativa ( $\mathrm{r}=0.554, p=0.017)$, ou seja, quanto mais idade, menor a fluência verbal fonológica.

A comparação do tempo de escolaridade à média dos TMF das vogais /a/, /i / e $/ \mathrm{u} /$, observa-se uma diferença significativa, sendo os maiores valores para com a maior escolaridade, não se encontrando referência na literatura que justificasse tal possibilidade.

Em Santos (2006), tem-se que a fluência verbal fonológica dos portadores de DP apresentam uma média de 16,63 pals./min. e, nos indivíduos sem perturbação neurológica, uma média de 24,40 pals./min. Os resultados do presente estudo quanto à fluência verbal fonológica contradizem os dados da literatura, pois os integrantes do grupo B, composto por participantes com grau de comprometimento III e IV obtiveram a média de palavras por minuto maior do que aqueles que se encontravam em estágios iniciais. Isso pode ser explicado pelo tempo de estudo do grupo $\mathrm{B}$ ser maior, em número de anos, que o do grupo A. 
Santos (2006) relata que os sujeitos com DP apresentam uma fluência verbal total inferior aos sujeitos sem a doença, o que se justifica pelo fato de os doentes estarem inseridos nos estágios iniciais (II e III) da doença, quando é observada pouca alteração nos níveis para com a fluência verbal. Essa é uma inferência possível, frente aos resultados do presente estudo.

Os resultados da fluência verbal semântica e fonológica verificados neste estudo, aliados aos dados da literatura (SANTOS, 2006; RODRIGUES; YAMASHITA; CHIAPPETTA, 2008; RIGON, 2010) evidenciam a importância do trabalho terapêutico para a manutenção das funções motoras e executivas junto aos indivíduos com a DP, considerando-se os graus de comprometimento da doença e o seu caráter degenerativo, para com o favorecimento da qualidade de vida dos parkinsonianos.

O grau de comprometimento da DP e os resultados da fluência verbal são indicativos da preservação das funções cognitivas lobares frontal e podem ser explicados pela manutenção desse grupo em atividades corporais e sociais. Esses resultados expõem as possibilidades de amenização da evolução da doença, a partir da inserção dos indivíduos com a DP em atividades motoras para as funções executivas, em ações sociais, retardando as manifestações degenerativas da DP ao máximo.

As atividades de manutenção das funções motoras e executivas contribuem para a qualidade de vida pela permanência social que possibilita, mantendo o indivíduo com a DP ativo e participador do curso da sua vida. A reabilitação ou a conservação de habilidades de cunho fonoterapêutico muito colaboram para essa permanência, quer pelo treinamento respiratório que contribui para uma maior mobilização torácico-abdominal e para o aumento da força muscular abdominal, com melhora da função respiratória e do aumento da intensidade vocal (ROSA; CIELO; CECHELLA, 2009), quer pelo restabelecimento da coaptação adequada das pregas vocais, da comunicação e da diminuição do risco para o comprometimento da função de deglutição.

\section{Conclusões}

O caráter degenerativo da DP compromete aspectos motores e cognitivos dos seus portadores ao longo da evolução da doença. A verificação da fluência verbal permite estabelecer a integridade das funções cerebrais. Os resultados obtidos com esse estudo são de uma fluência verbal semântica dos participantes mostrando-se abaixo do esperado, em se considerando a literatura disponível, e acima do verificado na literatura para a fluência fonológica. Os dados averiguados reforçam que o tempo de escolaridade influencia nos resultados da fluência verbal semântica e fonológica, visto que, quanto mais anos de estudo, melhor será a resposta para a fluência verbal, constatado pelo fato de quanto mais idoso for o indivíduo, menor será a resposta para a fluência verbal fonológica. Reforça-se a necessidade de inserção em atividades físicas, educacionais formais e de inserção social dos parkinsonianos, no intuito da melhoria da sua qualidade de vida. 


\section{Agradecimentos}

Aos associados da Apars, por sua disponibilidade e participação dos seus integrantes.

\section{The verbal fluency profile in people with Parkinson disease}

\section{Abstract}

The verbal fluency incorporates motor data's related to the time of phonation and the speed of speech, as well as of the aspects of the effectiveness of the language, from linguistics components, semantic and phonologic, in the establishment of its profile. The aim of this study is to verify the verbal fluency of a group of Parkinson's disease. It is a descritive study, cross-sectional and quantitative in which the subject-matter is the verbal fluency profile and its outcome, the Parkinson's disease. The sample was 18 persons from genders ( 9 women and 9 men), ages 48 to 78 years old and the average age being $65.5 \pm 8.4$ years old (SD 8.4 years). The time of phonation, glottis efficiency and verbal fluency tests had been verified. In the evaluating of the maximum phonation time (MPT), there was an average of 16.84 for vowels. The average s/z ratio was 0.84 and time of reading per minute of 130 words / min. In the semantic verbal fluency it was detected the average of 16 .words /min. and the phonologic verbal fluency the average of 17 .words / $\mathrm{min}$. The MPT was adequate to the variables age and gender. The semantic verbal fluency was lower than that reported in the literature and higher for phonologic verbal fluency. We reinforce the correlation between verbal fluency and years of schooling, as well as the group's cognitive preservation.

Keywords: Individuals with Parkinson's disease; Semantics; Language Tests.

\section{Referências}

ANDRADE, C. R. F. Fluência. In: ANDRADE, C. R. F.; BEFI-LOPES, D. M.; FERNANDES, F. D. M.; WERTZNER, H. F. $A B F W$ : teste de linguagem infantil nas áreas de fonologia, vocabulário, fluência e pragmática. Carapicuíba: Pró-Fono Departamento Editorial, 2000. p. 61-62.

AZEVEDO, L.; CARDOSO, F. Ação da levodopa e sua influência na voz e na fala de indivíduos com doença de Parkinson. Revista da Sociedade Brasileira de Fonoaudiologia, São Paulo, v. 14, n. 1, p. 136-141, 2009.

BEHLAU, M.; PONTES, P. A Avaliação da voz. In: BEHLAU, M.; PONTES, P. Avaliação e tratamento das disfonias. São Paulo: Editora Lovise. 1995. Cap. 3, p. 105-6.

BEHLAU, M.; MADAZIO, G.; FEIJÓ, D.; PONTES, P. Avaliação da voz. In: BEHLAU, M. A voz do especialista, v. I. Rio de Janeiro: Editora Revinter, 2001. Cap. 3. p. 85-245.

BRUNA, O.; SUBIRANA, J.; VILLALTA, V.; VIRGILI, C.; JUNQUÉ, C. Alteraciones neuropsicológicas y de la fluencia verbal en la Enfermedad de Parkinson. Rev. logop. foniatr. audiol., Barcelona, v. 28, n. 1, p. 8-14, Jan./Mar. 2008.

BUTMAN, J.; ALLEFRIR, F.; HARRIS, P.; DRAKE, M. Fluencia verbal en español datos normativos en Argentina. Medicina, Buenos Aires, v. 60, n. 5/1, p. 561-564, 2000.

CHANA, P.; JIMENEZ, M.; DIAZ, V.; JURI, C. Parkinson disease mortality rates in Chile. Rev. méd. Chile, Santiago de Chile, v. 141, n. 3, p. 327-331, mar. 2013.

DIAS, A.; LIMONGI, J. C. Tratamento nos distúrbios da voz na doença de Parkinson. Arq. Neuropsiquiatria, São Paulo, v. 61, n. 1 , p. 61-66, mar. 2003.

DUARTE, T.; CRENITTE, P.; LOPES-HERRERA, S. A. Caracterização dos indivíduos com distúrbios na fluência, atendidos na clínica - escola do curso de Fonoaudiologia da USP-Bauru. Revista CEFAC, São Paulo, v. 11, n. 3, p. 396-405, jul./set. 2009. 
FERREIRA, F. Características respiratórias, posturais e vocais na doença de Parkinson - estudo de casos. Dissertação [Mestrado em Distúrbios da Comunicação Humana] Universidade Federal de Santa Maria, 2008.

HOEHN, M. M.; YAHR, M. D. Parkinsonism: onset, progression, and mortality. Neurology, Minneapolis, v. 17, n. 5, p. 427-442, May 1967.

JAKUBOVICZ, R. A gagueira: teoria e tratamento de adultos e crianças. 5. ed. Rio de Janeiro: Editora Revinter, 1997, p. 3-10.

LIMONGI, J. Conhecendo melhor a doença de Parkinson: uma abordagem multidisciplinar com orientações práticas para o dia-a-dia. Rio de Janeiro: Editora Plexus, 2001, 117 p.

MARTINEZ-SANCHES, F. Transtornos del habla y la voz en la enfermedad de Parkinson. Revista de Neurologia, v. 51, p. 542-50, 2010.

MELO, L.; BARBOSA, E.; CARAMELLI, P. Declínio cognitivo e demência associados à doença de Parkinson: características clínicas e tratamento. Rev. Psiq. Clín., São Paulo, v. 34, n. 4, p. 176-83, dez. 2007.

MENESES, M.; TEIVE, H. Doença de Parkinson: aspectos clínicos e cirúrgicos. Rio de Janeiro: Editora Guanabara Koogan, 1996, 189 p.

MOURÃO, L. Intervenção Fonoaudiológica nos distúrbios do movimento. In: ORTIZ, K. Z. Distúrbios neurológicos adquiridos: fala e deglutição. 2. ed. São Paulo: Editora Manole, 2010. Cap. 8, p. 144-160.

MUNHOZ, R.P.; MOSCOVICH, M.; ARAUJO, P. D.; TEIVE, H.A.G. Movement disorders emergencies: a review. Arq. Neuro-Psiquiatr., São Paulo, v. 70, n. 6, p. 453-461, 2012.

NITRINI, R.; CARAMELLI, P.; BOTTINO, C.; DAMASCENO, B.; BRUCKI, S.; ANGHINAH, R. Diagnóstico de doença de Alzheimer no Brasil: avaliação cognitiva e funcional. São Paulo, Arq. Neuropsiquiatr, São Paulo, v. 63 , n. 3 , p. $720-7$, set. 2005 .

OLANOW, C. W.; STERN, M. B.; SETHI, K. The scientific and clinical basis for the treatment of Parkinson disease. Neurology, v. 72, n. 21,5.4, p. S1-S136, May 2009.
PALERMO, S.; BASTOS, I.; MENDES, M.; TAVARES, E.; SANTOS, D.; RIBEIRO, A. Avaliação e intervenção fonoaudiológica da doença de Parkinson: análise clínica-epidemiológica de 32 pacientes. Rev. Bras. Neurol., Rio de Janeiro, v. 45, n. 4, p. 17- 24, out./nov./dez. 2009.

PIOVEZAN, Mauro R. et al. Cognitive function assessment in idiopathic Parkinson's disease. Arq. Neuro-Psiquiatr., São Paulo, v. 65 , n. 4 , p. 942-6, Dec. 2007.

RIGON, B. O Perfil da fluência verbal em um grupo de idosos. (Monografia). Porto Alegre/ RS: Centro Universitário Metodista do IPA, 2010, 35p.

RODRIGUES, A.; YAMASHITA, E.; CHIAPPETTA, A. Teste de fluência verbal no adulto e no idoso: verificação da aprendizagem verbal. Revista CEFAC; São Paulo, v. 10, n. 4, p. 443-451, out./dez. 2008.

ROSA, J. C.; CIELO, C. A.; CECHELLA, C. Função fonatória em pacientes com Doença de Parkinson: uso de instrumento de sopro. Rev. CEFAC, São Paulo, v. 11, n. 2, p. 305-13, abr./jun. 2009.

SANTOS, J. A fluência verbal na doença de Parkinson. (Monografia) Porto: Universidade Fernando Pessoa, Faculdade de Ciências Humanas e Sociais, 2006. 96p.

SOUZA, R. L.; CARDOSO, M. C. A. F. Fluência e Prosódia: Aspectos diferenciais frente aos distúrbios. Rev Neurocienc, São Paulo, v. 21 , n. 3, p. 468-473, 2013.

VICCO, D.; SANTOS, S.; GONÇALVES, L. Análise acústica e perceptivo-auditiva da voz em pacientes parkinsonianos pré e pós-terapia fonoaudiológica. Cienc. Cuid. Saude, Maringá (PR), v. 8, n. 3, p.313-20, jul. /set. 2009.

VITORINO, M.; HOMEM, F. Doença de Parkinson: da fonação à articulação. Revista Fono Atual, São Paulo, v. 4, n. 17, p. 35-39, jul./set. 2001. 